\title{
The Intersection of HIV and Syphilis: Update on the Key Considerations in Testing and Management
}

\author{
Melody $\operatorname{Ren}^{1} \cdot$ Thomas Dashwood $^{1} \cdot$ Sharon Walmsley $^{1,2}$ (D) \\ Accepted: 18 May 2021 / Published online: 6 June 2021 \\ (C) The Author(s), under exclusive licence to Springer Science+Business Media, LLC, part of Springer Nature 2021
}

\begin{abstract}
Purpose of Review To highlight recent trends in the epidemiology of HIV and syphilis, the impact of the COVID epidemic, our approach to care of co-infected patients, and our views on important next steps in advancing the field.

Recent Findings HIV and syphilis co-infection has been on the rise in recent years although since the COVID pandemic there is a decrease in new diagnoses - it remains unclear if this represents a true decline or inadequate testing or under-reporting. Standard HIV care should include regular syphilis serology . Treatment and serological follow-up of syphilis in HIV positive and negative patients can be conducted similarly. Challenges remain in the diagnosis and management of neurosyphilis. New models for testing and prevention will be crucial next steps in controlling co-infection.

Summary The intersection of HIV and syphilis infections continues to pose new and unique challenges in diagnosis, treatment, and prevention.
\end{abstract}

Keywords HIV and syphilis · Syphilis testing · Syphilis treatment · Serology

\section{Introduction}

Our understanding of the interaction between syphilis and HIV continues to evolve and has increasing clinical importance given the rising rates of co-infection and the unique synergy between the two sexually transmittable infections. Syphilis facilitates the transmission and acquisition of HIV, and HIV has been documented to accelerate the natural history of syphilis [1-5]. Furthermore, HIV infection has been associated with false positive and negative syphilis serology complicating our diagnoses and management [6, 7]. Whether standard definitions of the serologic response and cure of syphilis after adequate therapy should be applied to those with HIV coinfection is unclear [8-10].

This article is part of the Topical Collection on Co-infections and Comorbidity

Sharon Walmsley

sharon.walmsley@uhn.ca

1 Division of Infectious Diseases, Department of Medicine, University of Toronto, Toronto, ON, Canada

2 Toronto General Hospital Research Institute, University Health Network, 200 Elizabeth Street, Room 13EN214,

Toronto, ON M5G2C4, Canada
The purpose of this review is to highlight recent trends in the epidemiology of HIV and syphilis, the impact of the COVID epidemic, our approach to care of co-infected patients, and our views on important next steps in advancing the field.

\section{Significant Trends in HIV and Syphilis Infection Rates}

\section{The Epidemiology of Syphilis and HIV}

The rates of HIV and syphilis co-infection globally have been on a meteoritic rise in recent years. In the late 1990s, the introduction of syndromic treatment for STIs, widespread sexual behavioral change, and sexual network disruption consequent to the HIV epidemic and fears of infection, contributed to a decreased prevalence in syphilis in many regions [11-14]. However, with the introduction of successful antiretroviral therapy and improved survival from HIV infection, and the demonstration of $\mathrm{U}=\mathrm{U}$ (undetectable =untransmittable), observed rates of syphilis have been increasing over the last two decades, most notably documented in the USA, Europe, Canada, Australia, and China [15-23]. The most recently available data indicate the number of cases in the USA have 
tripled from 2013 to 2018, have increased $~ 50 \%$ from 2009 to 2018 across Europe (the data influenced by the changing list of individual countries reporting statistics to the European CDC), have more than doubled from 2008 to 2017 in Canada, and have increased by $135 \%$ from 2013 to 2017 in Australia. Regional data from China demonstrates a general trend of increasing rates with some regions reporting higher rates than the USA.

In high-income countries, the re-emergence of syphilis is overwhelmingly disproportionate in men with a significant proportion self-identifying as MSM [24]. Data from 2017 and 2018 indicate that over $80 \%$ of infectious syphilis cases in the USA, Europe, Canada, and Australia were diagnosed in men.

In low-middle-income countries (LMIC), syphilis remains endemic in the general population, and accounts for over $90 \%$ of syphilis cases worldwide [25]. Although the MSM, transgender women, and sex worker populations remain at high risk in LMIC, data on the overall epidemiology is sparse due to under-reporting and may also be influenced by stigma and marginalization leading to limited access to health care [26]. Consequently, most epidemiological data in LMIC for syphilis are derived from World Health Organization (WHO) antenatal monitoring in pregnant women [27]. Rates of syphilis in pregnancy and congenital syphilis have increased in both high-income countries and LMIC, echoing the increase in primary and secondary syphilis in reproductive age women [28-30]. Globally, congenital syphilis is a major cause of neonatal and fetal mortality and the leading cause of stillbirth [31]. However, there has been some hope in this realm with five additional countries declared by the WHO to have successfully eliminated mother to child transmission of HIV and syphilis since 2015: Cuba, Thailand, Belarus, Armenia, and the Republic of Moldova [32, 33].

The reasons for the increase in syphilis rates are thought to be multi-factorial. Improved HIV management with highly effective anti-retroviral therapy (ART) has led to reestablishment of sexual networks and declines in previously adopted safer sex behaviors including unprotected sex [34-37]. The impact of condom-less sex has been further compounded by the introduction of pre-exposure prophylaxis (PrEP) and "serosorting" behaviors (selecting sexual partners based on concordant HIV serostatus) [36, 38-44]. Growing use of the Internet to find sexual partners has also been associated with increased high-risk sex including multiple sex partners and substance abuse during sex [45]. Moreover, syphilis prevalence is inversely proportional to the intensity of public health efforts and the current rise in syphilis has mirrored the decrease in public health funding in many jurisdictions [36, 37, 46, 47].

Increasing rates of syphilis across a wide variety of populations in diverse geographical settings demand that modernday clinicians have a heightened awareness of syphilis, especially in high-risk patients including those who are MSM or have risk factors for HIV acquisition.

\section{How Do We Define "Cure" of Syphilis in Those Co-infected with HIV}

Given our lack of ability to grow the organism, diagnosis and response to therapy is based on serology. HIV and syphilis management has been plagued by a lack of evidence to inform the expected response in syphilis serology after treatment in PLWH. An adequate serologic response after syphilis treatment is defined as a four-fold decrease in the RPR titer. This definition was adopted after mathematical models were used by Brown et al. to graph the decline in the Venereal Disease Research Laboratory test titers after treatment in 818 patients with primary and secondary syphilis [48]. The ongoing debate surrounds whether the time to the serologic response should differ in PLWH with infection in the various syphilis stages. There remains a wide variability among guidelines from the USA, Canada, European, and UK [49-52]. Many guidelines indicate that PLWH require "more time" to reach criteria for an adequate serologic response but do not delineate a specific timeline. The WHO guidelines on syphilis management do not comment on the expected timeline for serologic response post-treatment [53, 54]. Certainly, the imperfect nature of the current serologic methods for diagnosis complicates our ability to interpret testing after treatment.

Defining serologic cure on indirect measures of disease activity continues to challenge health care providers who base clinical decision making on those tests. This is clouded by rates of syphilis re-infection, loss to follow-up, and serofast states, whereby titers remain low indefinitely, when some recommend consideration of re-treatment for syphilis $[10,55]$. Further confounding factors include the variability in HIV status including viral load, CD4 counts, and the evolving environment of treatment with increasingly efficacious antiretroviral therapies [56]. An improved understanding of the serological response after adequate treatment in PLWH is critical in defining management.

To try to understand this in the real-world setting, we undertook an evaluation of the serologic response to syphilis in 171 persons co-infected with HIV in our tertiary care clinic. We demonstrated that over $90 \%$ of our patients across all syphilis stages achieved an adequate serological response after appropriate treatment as defined by the 2015 CDC guidelines. It remains unclear how to monitor or manage the remaining $10 \%$ [57].

\section{Drug Supply Issues and Macrolide Resistance in Syphilis}

First-line standard therapy for syphilis is treatment with penicillin or doxycycline. Barriers to using penicillin include the need for injections, drug shortages, and allergies while 
doxycycline use is not recommended in pregnant women. The appropriate management for syphilis is additionally complicated by the emergence and proliferation of macrolide resistant strains.

Benzathine penicillin G (BPG) is off patent with a market price of pennies per dose but is expensive to manufacture as a sterile injectable medication. Several pharmaceutical manufacturers have stopped producing BPG due to these economics [58]. From 2014 to 2016, the WHO collected country level data and found 39 of 95 responding countries and territories were experiencing BPG shortage, including in high syphilis morbidity regions [58]. Brazil notably had an increase in congenital syphilis cases from 4.0 per 1000 live births in 2012 to 6.5 per 1000 live births in 2015 after a manufacturer ended production on 2013. In Rio de Janerio, the BPG shortage was associated with a 2.23-fold increase in the risk of congenital syphilis [58, 59]

Azithromycin can be used in patients at high risk for STIs to symptomatically treat for gonorrhea and chlamydia and in the 1990s was a simple one dose alternative treatment to penicillin for syphilis treatment [60]. The first cases of azithromycin clinical failure were described shortly after in the early 2000s. Retrospective analyses in Canada and the USA have shown the proportions of samples containing one of the two single nucleotide polymorphisms responsible for conferring resistance, A2058G and A2059G, have increased over time [61-65]. To date, macrolide-resistant syphilis has been described in Australia, Canada, China, Europe, and the USA with some regions reporting macrolide resistance in over $80-90 \%$ of clinical isolates [26, 66-68]. This has eliminated azithromycin as a second-line option for syphilis treatment in some geographical regions and has caused very cautious use elsewhere [69].

There is ongoing pressure for international co-operation to ensure adequate supply of penicillin for syphilis treatment in addition to judicious monitoring of the effectiveness of alternate, less studied non-penicillin antimicrobials.

\section{COVID-19, HIV, and Syphilis}

Since the start of the COVID-19 global pandemic, the impact on the management of patients with HIV and syphilis has been closely monitored.

To date there has been no evidence that PLWH hospitalized for COVID-19 fair any worse when compared to similar demographic groups [70, 71]. However, there have been increasing concerns about the effects of social distancing and lockdown measures on maintaining care for chronic HIV and STI management. This includes ongoing prevention and treatment efforts in socially and economically vulnerable populations who are at high risk of COVID-19 and HIV, ensuring minimal disruptions to regular HIV care and antiretroviral therapy access, and juggling limited resources in over- burdened health systems as priorities shift towards the management of COVID-19 [72, 73]. Modelling studies in high burden HIV settings have shown dire consequences should HIV care and prevention work fall to the wayside; deaths due to HIV could increase by up to $10 \%$ and a 6 -month interruption of ARV could lead to more than 500,000 more deaths from AIDS-related illnesses in sub-Saharan Africa in 2020 2021 compared to the 470,000 AIDS-related deaths in the region in 2018 [74, 75]. Survey data has indicated that COVID-19 has prevented patients from accessing their chronic medications [75].

Social distancing and lockdown measures have led to decreased rates of diagnosis of syphilis in Rome and Madrid and syphilis and HIV in Taiwan compared to same time periods in previous years [76-88]. This phenomenon has also been noted in public health reports comparing 2020 data for HIV and syphilis in regions in Canada and in the USA [79, 80]. It is unclear if the reduction is due to less frequent sexual encounters and a true decline in syphilis and HIV rates or if patients are postponing diagnosis and care leading to under-reporting as seen in other specialties. Encouraging people to seek care for STIs during the COVID-19 pandemic continues to be important. It remains to be seen if COVID-19 has any persistent substantial effect on STI epidemiology, whether that is a decrease in rates due to disrupted sexual networks, an increase in rates due to lack of prevention and treatment of STIs, or no effect at all.

As the COVID-19 global pandemic continues, we must be vigilant and ensure that prevention and health care services for HIV and syphilis remain accessible and be flexible in meeting patients' needs in this unprecedented time.

\section{Some Personal Observations on HIV and Syphilis}

Similar to the international and Canadian trends, our HIV clinic in a tertiary care center in Toronto, Canada, has experienced an increase in syphilis diagnoses among our patient population. From 2000 to 2008, the average number of patients diagnosed with syphilis was $11.3 /$ year. For the total number of patients seen in our clinic, this translated to 1413 cases/100,000 population compared to the national Canadian rate of 4.2 cases $/ 100,000$ population in 2008 [81]. From 2009 to 2017 , there was an average of 38.8 patients diagnosed with syphilis each year translating to 3527 cases/100,000 population compared to the national Canadian rate of 79.5 cases/ 100,000 population in 2017 [81]. From 2000 to 2017, there was a $250 \%$ increase in syphilis diagnosis in our clinic. In our study of the serologic response after syphilis treatment, we observed that $17.5 \%$ in our included cohort had neurosyphilis compared to $9.4 \%$ with primary, $31 \%$ with secondary, $15.2 \%$ with early latent, and $26.9 \%$ with late latent [57]. This may be explained by the tertiary nature of our center; our patient 
population exclusively includes PLWH who may be at higher risk for neurosyphilis $[82,83]$.

\section{Our Approach to Caring for Patients Co-infected with HIV and Syphilis}

In our HIV clinic, syphilis serology has become a part of the standard panel of bloodwork completed on patients during each visit since 2018 when our research demonstrated this to be an effective approach [84]. Previous studies have also indicated that in our context implementing routine syphilis testing is cost effective and supported by patients $[85,86]$. We diagnose and monitor patients for syphilis based on the 2015 Centre of Disease Control guidelines for syphilis management and have described that over $90 \%$ of our patients across all syphilis stages achieved an adequate serological response after appropriate treatment $[57,86]$. Our first-line therapy is benzathine penicillin for primary, secondary, early latent, and late latent syphilis and IV penicillin G for neurosyphilis with only $4 \%$ of patients receiving doxycycline for documented or reported penicillin allergy [57].

\section{Our Approach to Controversial Topics: Neurosyphilis}

Diagnosing and managing neurosyphilis in PLWH presents several challenges, including the diagnosis and management of asymptomatic neurosyphilis (ANS) and limited evidence to guide optimal therapy.

There is ongoing controversy as to whether ANS is a true entity, and whether it constitutes an increased risk of neurological complications if left untreated. Asymptomatic neurosyphilis in PLWH became a concern after several case reports were published during the height of the HIV epidemic, showing that patients who had been treated for syphilis with conventional regimens later developed "neurorecurrence" of the disease [87-90]. However, there is no unifying definition for ANS, which is typically defined based on specific cerebrospinal fluid (CSF) abnormalities. Regardless, these reports spurred clinicians to consider whether PLWH who are diagnosed with syphilis should undergo lumbar puncture (LP) routinely to assess the CSF. Investigators then attempted to risk-stratify patients in order to determine who would benefit most from CSF examination. Reliable criteria for determining this risk have not been established, and a consensus on which patients should undergo examination for neurosyphilis remains elusive. The European guidelines on syphilis, updated in 2020, state that CSF assessment is not indicated in early syphilis in the absence of neurological symptoms, and regardless of HIV status [51]. Furthermore, despite benzathine penicillin $\mathrm{G}$ not reaching reputed treponemicidal levels in the CSF, a recent study by Tomkins et al. showed that among 64 PLWH who were deemed "high risk" for the development of symptomatic neurosyphilis ( $\mathrm{RPR} \geq 1: 32$, and/or CD4 $<350$ / $\mathrm{mm}^{3}$ ), and treated with a single dose of intermuscular benzathine penicillin, one participant developed ANS, and none developed symptomatic neurosyphilis [91]. Despite this, some experts continue to recommend CSF assessment in asymptomatic patients to exclude neurosyphilis in the following circumstances: in PLWH who are diagnosed with late syphilis and have CD4 cells $\leq 350 / \mathrm{mm}^{3}$ and/or a serum RPR titer $>1: 32$, in those who have serologic failure, or in those given non-penicillin therapy for late syphilis [92].

Our practice is consistent with the European guidelines in that we do not conduct CSF assessments in patients without neurologic symptoms. In patients who have an inadequate response to syphilis therapy, we offer to conduct an LP, or to treat for presumptive neurosyphilis. Similarly, in cases where serologic testing raises the possibility of neurosyphilis, and a patient has vague or non-specific symptoms, we engage in a discussion of the risk and benefits of conducting an LP, treating for neurosyphilis presumptively, or treating for late syphilis, which many argue is adequate therapy for neurosyphilis, with frequent reassessment and the option to escalate investigations if necessary. In terms of assessing response to therapy, we do not routinely repeat an LP, and instead we rely on serological response as a strong predictor of CSF response, as previously demonstrated [93, 94].

The challenges described above in syphilis therapy are exacerbated in neurosyphilis due to a reliance on penicillinbased therapies. Intravenous aqueous penicillin has long been the mainstay of therapy; despite few studies to support its use, we know it reaches treponemicidal concentrations in the CSF, and has been a reliable therapy for decades. Intramuscular procaine penicillin, administered with probenecid, is also a recommended alternative regimen by the CDC and European guidelines $[50,51]$, and a recent study by Dunaway et al. suggested it is equivalent to intravenous aqueous penicillin G, regardless of HIV status [95]. Ceftriaxone is a common alternative regimen that can be used in most people with penicillin allergy, but a recent Cochrane review identified minimal evidence to support its use [96]. While other drugs such as doxycycline and azithromycin have been used for syphilis therapy, and others, such as cefixime [97], are being investigated, none has been evaluated for neurosyphilis specifically, leaving us with few alternative therapeutic options. Given the intensive resource requirements of intravenous therapy, and penicillin desensitization when required, evaluating alternative agents will be increasingly important as syphilis rates continue to soar.

\section{Future Directions: What We Need to Provide Better Care for Patients}

The current landscape in HIV and syphilis co-infection has two major areas of improvement that could help advance 
clinical management: improved testing and diagnostic methods for syphilis and increased efforts towards prevention of HIV and syphilis.

\section{Testing}

HIV diagnostic testing was recognized as a crucial contributor to the medical and public health response to the HIV epidemic. Since 1985, when the US Food and Drug Administration (FDA) approved the first HIV detection systems, the evolution in laboratory technologies led to the development of the highly sensitive screening test and highly specific confirmatory assay used today. The improvement of HIV diagnostics has revolutionized the management and prevention of HIV.

Contrary to HIV, there remains ample opportunity for improvement in syphilis testing to minimize morbidity, mortality, and transmission. Traditional darkfield microscopy is no longer routinely available and current serologic tests rely on the patient humoral immune response to diagnose syphilis. These indirect measures of syphilis are fraught with issues in interpretation. Rapid testing is available but is limited to treponemal antibody results and does not differentiate between active and previously treated infection [53]. The 2016 WHO syphilis guidelines suggest using the rapid treponemal antibody assay in antenatal screening in high-burden areas as the turnaround time is $10-15 \mathrm{~min}$ and the assay does not require refrigerated storage or lab equipment. Reported sensitivity and specificity range from 85 to $98 \%$ and 93 to $99 \%$, respectively, compared to the TPPA as the reference standard. However, isolated treponemal antibody may lead to unnecessary treatment and stigma associated with syphilis diagnosis. Rapid testing combining non-treponemal and treponemal antibodies has been researched but not sufficiently validated or field tested and is not recommended in 2016 WHO syphilis guideline [98]. No combination rapid tests are currently available in the USA or Canada. PCR testing for syphilis is also an ongoing area of interest. There are various types of PCR testing; however, none are approved by the FDA and PCR testing for syphilis remains costly [99]. PCR is best for detecting primary syphilis with swabs from chancres although it would amplify both live and dead organisms. There is conflicting data regarding the sensitivity of PCR in secondary syphilis, and it is not felt to be suitable for screening asymptomatic individuals as PCR sensitivity drops to $24-32 \%$ in blood and CSF $[99,100]$.

\section{Prevention}

The resurgence of syphilis has spurred interest in expanding syphilis prevention methods beyond traditional measures. Investigation into pharmacologic prevention strategies has increased recently with the advent of pre-exposure prophylaxis (PrEP) for HIV. Due to observations of high incidences of bacterial STIs in several HIV PrEP studies, post-exposure prophylaxis (PEP) for syphilis was investigated in the openlabel phase of the ANRS IPERGAY trial for HIV PrEP [101]. This sub-study of 232 participants showed that taking $200 \mathrm{mg}$ of oral doxycycline within $72 \mathrm{~h}$ of condomless sex (without exceeding $600 \mathrm{mg}$ total per week) reduced the incidence of syphilis and chlamydia, with a hazard ratio of $0.30(95 \% \mathrm{CI}$ : $0.13-0.70, p=0.006)$ and 0.27 (95\% CI: 0.07-0.98, p<0.05) respectively. Further studies are ongoing to investigate the role of doxycycline as syphilis PrEP, such as in the Dual Daily HIV and Syphilis Pre-exposure prophylaxis trial [102]. Preliminary results have been promising. In patients using HIV PrEP, taking doxycycline $100 \mathrm{mg}$ orally daily immediately compared to deferring doxycycline (starting by 24 weeks) resulted in a syphilis incidence of 0 compared to 8.74 per 100 person years, and a chlamydia incidence of 0 versus 69.9 infections per 100 person years. Another pilot study was able to show reduced STIs in participants taking doxycycline $100 \mathrm{mg}$ daily (versus a contingency management strategy with financial incentives to avoid STIs), but not in syphilis specifically [103]. In addition, a systematic review of studies assessing the efficacy of periodic presumptive treatment for bacterial STIs among sex workers showed efficacy for reducing the incidence of chlamydia, gonorrhea, and ulcerative STIs, but not syphilis [104]. Further evidence is required to support the use of PEP, PrEP, and presumptive treatment strategies for syphilis and other bacterial STIs, and investigations into the possible unintended effects of these prevention strategies, such as antimicrobial resistance, or Clostridium difficile superinfection will be important.

An effective and accessible vaccine for syphilis could turn the tide on syphilis globally. Recently, Lithgow et al. identified a promising vaccine target, Tp0751, a vascular adhesin implicated in the dissemination of Treponema pallidum [105]. They have shown efficacy in animal models, but efficacy and safety in humans is yet to be shown. While a vaccine is likely years away from discovery, it could have a significant impact on curbing syphilis worldwide.

Integrating HIV care with syphilis prevention and care is crucial for several reasons, especially with the advent of PrEP for HIV. We know that HIV and syphilis can increase the risk of becoming infected with the other [103], and that certain populations are at high risk for both infections. Therefore, anyone presenting with a new diagnosis of HIV should be screened for syphilis, and vice versa. Furthermore, the guidelines for HIV PrEP in Canada and the USA now consider a new diagnosis of syphilis as an indication to consider starting HIV PrEP [106, 107]. In addition, frequent healthcare contact for PLWH and patients using PrEP could theoretically lead to increased rates of STI screening, which may result in earlier diagnosis of STIs, earlier treatment, and reduced onward transmission. 
While this has not been shown definitively, there is growing data to suggest this is possible [108].

Other novel strategies for improving STI screening rates include home testing kits for asymptomatic individuals, such as used by Sexual Health London in the United Kingdom [109], and coupling syphilis screening serology with other routine tests, such as viral loads for PLWH [84]. Adapting these various tools and strategies to the needs of local communities worldwide is crucial to preventing both syphilis and HIV.

\section{Conclusion}

The intersection of HIV and syphilis infections continues to evolve in the modern era, posing new and unique challenges in diagnosis, treatment, and prevention. Here we have explored the impacts of rising syphilis rates globally, the advent of HIV PrEP, and the COVID-19 pandemic. In addition, we have outlined our approach to caring for the patient with both HIV and syphilis. Moving forward, there continues to be a significant and growing need for more accessible testing strategies, alternative treatment regimens, and improved approaches to the prevention of both HIV and syphilis.

\section{Declarations}

Conflict of Interest Melody Ren and Thomas Dashwood declare that they have no conflict of interest. Sharon Walmsley reports grants, personal fees, non-financial support, and other from Merck; grants, personal fees, non-financial support, and other from Gilead Sciences; grants, personal fees, non-financial support, and other from ViiV Healthcare; grants, personal fees, non-financial support, and other from GSK; and grants and personal fees from Janssen, outside the submitted work.

Human and Animal Rights and Informed Consent This article does not contain any studies with human or animal subjects performed by any of the authors.

\section{References}

1. French, Patrick. "Syphilis." BMJ, 2007, doi:https://doi.org/10. 1136/bmj.39085.518148.BE, Syphilis.

2. Sellati TJ, et al. Virulent Treponema Pallidum, lipoprotein, and synthetic lipopeptides induce CCR5 on human monocytes and enhance their susceptibility to infection by human immunodeficiency virus type 1 . The Journal of Infectious Diseases. 2000;181(1):283-93. https://doi.org/10.1086/315209.

3. Buchacz K, et al. Syphilis increases HIV viral load and decreases CD4 cell counts in HIV-infected patients with new syphilis infections. AIDS. 2004;18(15):2075-9. https://doi.org/10.1097/ 00002030-200410210-00012.

4. Kofoed K, et al. Syphilis and human immunodeficiency virus (HIV)-1 coinfection: influence on CD4 T-cell count, HIV-1 viral load, and treatment response. Sexually Transmitted Diseases.
2006;33(3):143-8. https://doi.org/10.1097/01.olq.0000187262. 56820.c0.

5. Lynn WA, Lightman S. Syphilis and HIV: a dangerous combination. The Lancet Infectious Diseases. 2004;4(7):456-66. https:// doi.org/10.1016/s1473-3099(04)01061-8.

6. Smith G, Holman RP. The prozone phenomenon with syphilis and HIV-1 co-infection. Southern Medical Journal. 2004;97(4):379 82. https://doi.org/10.1097/01.smj.0000121204.58881.60.

7. Geusau A, et al. Biological false-positive tests comprise a high proportion of venereal disease research laboratory reactions in an analysis of 300,000 sera. International Journal of STD \& AIDS. $2005 ; 16(11): 722-6$. https://doi.org/10.1258/ 095646205774763207.

8. Rolfs RT, et al. A randomized trial of enhanced therapy for early syphilis in patients with and without human immunodeficiency virus infection. New England Journal of Medicine. 1997;337(5): 307-14. https://doi.org/10.1056/nejm199707313370504.

9. Ghanem KG, et al. Serological response to syphilis treatment in HIV-positive and HIV-negative patients attending sexually transmitted diseases clinics. Sexually Transmitted Infections. 2006;83(2):97-101. https://doi.org/10.1136/sti.2006.021402.

10. Malone JL, et al. Syphilis and neurosyphilis in a human immunodeficiency virus type-1 seropositive population: evidence for frequent serologic relapse after therapy. The American Journal of Medicine. 1995;99(1):55-63. https://doi.org/10.1016/s00029343(99)80105-3.

11. Pettifor A, et al. How effective is syndromic management of STDs? Sexually Transmitted Diseases. 2000;27(7):371-85. https://doi.org/10.1097/00007435-200008000-00002.

12. Johnson LF, et al. The effect of syndromic management interventions on the prevalence of sexually transmitted infections in South Africa. Sexual \& Reproductive Healthcare. 2011;2(1):13-20. https://doi.org/10.1016/j.srhc.2010.08.006.

13. Chesson HW, et al. AIDS mortality may have contributed to the decline in syphilis rates in the United States in the 1990s. Sexually Transmitted Diseases. 2003;30(5):419-24. https://doi.org/10. 1097/00007435-200305000-00008.

14. Kenyon CR, et al. What underpins the decline in syphilis in Southern and Eastern Africa? An Exploratory Ecological Analysis. International Journal of Infectious Diseases. 2014;29: 54-61. https://doi.org/10.1016/j.ijid.2014.05.014.

15. Abara WE, et al. Syphilis trends among men who have sex with men in the United States and Western Europe: a systematic review of trend studies published between 2004 and 2015. PLOS ONE. 2016;11(7). https://doi.org/10.1371/journal.pone.0159309.

16. European Centre for Disease Prevention and Control. "Syphilis. In: ECDC. Annual epidemiological report for 2018." Stockholm: ECDC; 2020. https://www.ecdc.europa.eu/sites/default/files/ documents/syphilis-aer-2018.pdf

17. Australian Government Department of Health. "Third national sexually transmissible infections strategy 2014-2017. “ 2014. Australian Government Department of Health.https:// consultations.health.gov.au/ohpd-health-protection-policybranch/test-national-strategies-for-blood-borne-viruses-a/ supporting documents/Draft $\% 203$ rd $\% 20$ National $\% 20$ STI\% 20Strategy\%2020142017\%20D14818806.PDF

18. Australian Government Department of Health. "The health of Australia's males, sexual health." Australian Institute of Health and Welfare, (n.d.) www.aihw.gov.au/reports/men-women/malehealth/contents/how-healthy/sexual-health.

19. Chen G, et al. Syphilis incidence among men who have sex with men in China: results from a meta-analysis. International Journal of STD \& AIDS. 2016;28(2):170-8. https://doi.org/10.1177/ 0956462416638224

20. Centers for Disease Control and Prevention. "2018 sexually transmitted diseases surveillance: syphilis." Atlanta, GA: Centers for 
Disease Control and Prevention; 2019, Available at https://www. cdc.gov/std/stats18/syphilis.htm

21. Public Health Agency of Canada. 'Government of Canada.' "CCDR: Volume 45 - 11 - November 7, 2019: sexually transmitted infections in men who have sex with men - Canada." Ottawa, Canada: Public Health Agency of Canada. Government of Canada; 2019, Available at www.canada.ca/en/public- health/ services/reports-publications/canada-communicable- diseasereport-ccdr/monthly-issue/2019-45/issue-11-novem- ber-7-2019. html

22. Wong NS, et al. Distribution of reported syphilis cases in South China: spatiotemporal analysis. Scientific Reports. 2018;8(1). https://doi.org/10.1038/s41598-018-27173-y.

23. Yang S, et al. Epidemiological features of and changes in incidence of infectious diseases in China in the first decade after the SARS outbreak: an observational trend study. The Lancet Infectious Diseases. 2017;17(7):716-25. https://doi.org/10.1016/ s1473-3099(17)30227-x.

24. Bibbins-Domingo K, et al. Screening for syphilis infection in nonpregnant adults and adolescents. JAMA. 2016;315(21):2321. https://doi.org/10.1001/jama.2016.5824.

25. Hook EW, Peeling RW. Syphilis control - a continuing challenge. New England Journal of Medicine. 2004;351(2):122-4. https://doi.org/10.1056/nejmp048126.

26. Kojima N, Klausner JD. An update on the global epidemiology of syphilis. Current Epidemiology Reports. 2018;5(1):24-38. https:// doi.org/10.1007/s40471-018-0138-z.

27. World Health Organization. Global Health Observatory data repository. 2017. Available at: http://apps.who.int.myaccess.library. utoronto.ca/gho/data/node.main.A1357STI?lang=en.

28. Centers for Disease Control and Prevention. Congenital syphilis-United States, 2003-2008. MMWR Morb Mortal Wkly Rep. 2010;59(14):413-7.

29. Patton ME, et al. Primary and secondary syphilis - United States, 2005-2013. MMWR Morb Mortal Wkly Rep. 2014;63(18):4026.

30. Lithgow KV, Cameron CE. Vaccine development for syphilis. Expert Review of Vaccines. 2016;16(1):37-44. https://doi.org/ 10.1080/14760584.2016.1203262.

31. Newman L, et al. Global estimates of syphilis in pregnancy and associated adverse outcomes: analysis of multinational antenatal surveillance data. PLoS Medicine. 2013;10(2). https://doi.org/10. 1371/journal.pmed.1001396.

32. Ishikawa $\mathrm{N}$, et al. Elimination of mother-to-child transmission of hiv and syphilis in Cuba and Thailand. Bulletin of the World Health Organization. 2016;94(11). https://doi.org/10.2471/blt.16. 185033.

33. "WHO Validation for the Elimination of Mother-to-Child Transmission of HIV and/or Syphilis." World Health Organization, World Health Organization, 2 Apr. 2020, www. who.int/reproductivehealth/congenital-syphilis/WHO-validationEMTCT/en/.

34. Kenyon CR, et al. The global epidemiology of syphilis in the past century - a systematic review based on antenatal syphilis prevalence. PLOS Neglected Tropical Diseases. 2016;10(5). https://doi. org/10.1371/journal.pntd.0004711.

35. Kenyon CR, et al. Syphilis reinfections pose problems for syphilis diagnosis in Antwerp, Belgium - 1992 to 2012. Eurosurveillance. 2014;19(45). https://doi.org/10.2807/1560-7917.es2014.19.45. 20958.

36. Clement ME, Hicks CB. Syphilis on the rise. JAMA. 2016;315(21):2281-3. https://doi.org/10.1001/jama.2016.7073.

37. Mayor S. Syphilis and Gonorrhoea increase sharply in England. BMJ. 2015;350, no. jun25 12. https://doi.org/10.1136/bmj.h3457.

38. Kojima N, et al. Pre-exposure prophylaxis for HIV infection and new sexually transmitted infections among men who have sex with men. AIDS. 2016;30(14):2251-2. https://doi.org/10.1097/ qad.0000000000001185.

39. Liu AY, et al. Preexposure prophylaxis for HIV infection integrated with municipal- and community-based sexual health services. JAMA Internal Medicine. 2016;176(1):75. https://doi.org/10. 1001/jamainternmed.2015.4683.

40. Volk JE, et al. No new HIV infections with increasing use of HIV preexposure prophylaxis in a clinical practice setting. Clinical Infectious Diseases. 2015;61(10):1601-3. https://doi.org/10. 1093/cid/civ778.

41. La Fata L, et al. High asymptomatic STI prevalence and incidence in French MSM starting PrEP. San Diego: IDWeek. 2017;2017.

42. Lal L, et al. Medication adherence, condom use and sexually transmitted infections in Australian preexposure prophylaxis users. AIDS. 2017;31(12):1709-14. https://doi.org/10.1097/qad. 0000000000001519

43. Scott HM, Klausner JD. Sexually transmitted infections and preexposure prophylaxis: challenges and opportunities among men who have sex with men in the US. AIDS Research and Therapy. 2016;13(1). https://doi.org/10.1186/s12981-016-0089-8.

44. Traeger MW, et al. Association of HIV preexposure prophylaxis with incidence of sexually transmitted infections among individuals at high risk of HIV infection. JAMA. 2019;321(14):1380. https://doi.org/10.1001/jama.2019.2947.

45. Simon Rosser BR, Oakes JM, Horvath KJ, Konstan JA, Danilenko GP, Peterson JL. HIV sexual risk behavior by men who use the internet to seek sex with men: results of the Men's INTernet Sex Study-II (MINTS-II). AIDS and Behavior. 2009;13(3):488-98. https://doi.org/10.1007/s10461-009-9524-3.

46. Gulland A. Number of cases of syphilis continue to rise: BMJ; 2017. https://doi.org/10.1136/bmj.j2807.

47. McCarthy M. Syphilis rate rises in the US. BMJ. 2014;349, no. dec19(9). https://doi.org/10.1136/bmj.g7756.

48. Brown ST, Zaidi A, Larsen SA, Reynolds GH. Serological response to syphilis treatment. A new analysis of old data. JAMA. 1985;253(9):1296-9.

49. Public Health Agency of Canada. "Syphilis - Section 5 Management and treatment of specific infections - Canadian guide- lines on sexually transmitted infections." Ottawa, Canada: Public Health Agency of Canada; 2016, Available at http://www.phac- aspc.gc.ca/std-mts/sti-its/cgsti-ldcits/section-510-eng.php

50. Centers for Disease Control and Prevention. "Syphilis." Atlanta, GA: Centers for Disease Control and Prevention; 2016, Available at www.cdc.gov/std/tg2015/syphilis.htm

51. Janier M, Unemo M, Dupin N, Tiplica GS, Potočnik M, Patel R. 2020 European guideline on the management of syphilis. Journal of the European Academy of Dermatology and Venereology. 2020;35:574-88. https://doi.org/10.1111/jdv.16946.

52. Kingston M, French P, Higgins S, McQuillan O, Sukthankar A, Stott C, et al. UK National Guidelines on the Management of Syphilis 2015. International Journal of STD \& AIDS. 2015;27(6):421-46. https://doi.org/10.1177/0956462415624059.

53. WHO guidelines for the treatment of Treponema pallidum (syphilis) - 2016, https://apps.who.int/iris/bitstream/handle/ 10665/249572/9789241549806-eng.pdf;jsessionid= 513ECEDA51D97FC0FCD7DECC8E7CA783? sequence=1.

54. WHO guidelines for the management of sexually transmitted infections - 2004, https://apps.who.int/iris/bitstream/handle/10665/ 42782/9241546263_eng.pdf?sequence=1

55. Zetola NM, Klausner JD. Syphilis and HIV infection: an update. Clinical Infectious Diseases. 2007;44(9):1222-8. https://doi.org/ 10.1086/513427.

56. Farhi D, Dupin N. Management of syphilis in the HIV-infected patient: facts and controversies. Clinics in Dermatology. 
2010;28(5):539-45. https://doi.org/10.1016/j.clindermatol.2010. 03.012 .

57. Ren M, et al. Deciphering the Serological Response to Syphilis Treatment in Men Living with HIV. AIDS. 2020;34(14):2089-96. https://doi.org/10.1097/qad.0000000000002656.

58. Nurse-Findlay $\mathrm{S}$, et al. Shortages of benzathine penicillin for prevention of mother-to-child transmission of syphilis: an evaluation from multi-country surveys and stakeholder interviews. PLOS Medicine. 2017;14(12). https://doi.org/10.1371/journal.pmed. 1002473.

59. Ueleres BJ, et al. The shortage of benzathine penicillin and its impact on congenital syphilis incidence: an ecologic study in the City of Rio De Janeiro: Clinical Infectious Diseases; 2020. https:// doi.org/10.1093/cid/ciaa1716.

60. Stamm LV, Bergen HL. A point mutation associated with bacterial macrolide resistance is present in both 23S RRNA genes of an erythromycin-resistant Treponema pallidum clinical isolate. Antimicrobial Agents and Chemotherapy. 2000;44(3):806-7. https://doi.org/10.1128/aac.44.3.806-807.2000.

61. Katz KA, Klausner JD. Azithromycin resistance in Treponema Pallidum. Current Opinion in Infectious Diseases. 2008;21(1): 83-91. https://doi.org/10.1097/qco.0b013e3282f44772.

62. Stamm LV. Syphilis: antibiotic treatment and resistance. Epidemiology and Infection. 2014;143(8):1567-74. https://doi. org/10.1017/s0950268814002830.

63. Stamm LV. Global challenge of antibiotic-resistant Treponema Pallidum. Antimicrobial Agents and Chemotherapy. 2009;54(2): 583-9. https://doi.org/10.1128/aac.01095-09.

64. Lukehart SA, et al. Macrolide resistance in Treponema Pallidumin the United States and Ireland. New England Journal of Medicine. 2004;351(2):154-8. https://doi.org/10.1056/nejmoa040216.

65. Beale MA, et al. Genomic epidemiology of syphilis reveals independent emergence of macrolide resistance across multiple circulating lineages. Nature Communications. 2019;10(1). https://doi. org/10.1038/s41467-019-11216-7.

66. A2058G Prevalence Workgroup. Prevalence of the 23S rRNA A2058G point mutation and molecular subtypes in Treponema pallidum in the United States, 2007 to 2009. Sex Transm Dis. 2012;39(10):794-8. https://doi.org/10.1097/OLQ. 0b013e31826f36de.

67. Chen X-S, Yin YP, Wei WH, Wang HC, Peng RR, Zheng HP, et al. High prevalence of azithromycin resistance to Treponema Pallidum in geographically different areas in China. Clinical Microbiology and Infection. 2013;19(10):975-9. https://doi.org/ 10.1111/1469-0691.12098.

68. Centers for Disease Control and Prevention. "Procaine penicillin G shortage.” 2017. Available at: https://www-cdc-gov.myaccess. library.utoronto.ca/std/treatment/drugnotices/procaine-peng.htm.)

69. Stamm LV. Syphilis: re-emergence of an old foe. Microbial Cell. 2016;3(9):363-70. https://doi.org/10.15698/mic2016.09.523.

70. Sigel K, et al. Coronavirus 2019 and people living with human immunodeficiency virus: outcomes for hospitalized patients in New York City. Clinical Infectious Diseases. 2020;71(11):29338. https://doi.org/10.1093/cid/ciaa880.

71. Karmen-Tuohy S, et al. Outcomes among HIV-positive patients hospitalized with COVID-19. JAIDS Journal of Acquired Immune Deficiency Syndromes. 2020;85(1):6-10. https://doi. org/10.1097/qai.0000000000002423.

72. Lodge W, Kuchukhidze S. COVID-19, HIV, and migrant workers: the double burden of the two viruses. AIDS Patient Care and STDs. 2020;34(6):249-50. https://doi.org/10.1089/apc. 2020.0092

73. Jiang H, et al. Maintaining HIV care during the COVID-19 pandemic. The Lancet HIV. 2020;7(5). https://doi.org/10.1016/ s2352-3018(20)30105-3.
74. Hogan AB, et al. Potential impact of the COVID-19 pandemic on $\mathrm{HIV}$, tuberculosis, and malaria in low-income and middle-income countries: a modelling study. The Lancet Global Health. 2020;8(9). https://doi.org/10.1016/s2214-109x(20)30288-6.

75. Jewell BL, et al. Potential effects of disruption to HIV programmes in Sub-Saharan Africa caused by COVID-19: results from multiple mathematical models. The Lancet HIV. 2020;7(9). https://doi.org/10.1016/s2352-3018(20)30211-3.

76. Latini A, et al. Is COVID-19 affecting the epidemiology of STIs? The experience of syphilis in Rome. Sexually Transmitted Infections. 2020;97(1):78. https://doi.org/10.1136/sextrans-2020054543.

77. Chia, Chun-Chieh, et al. "Diagnoses of syphilis and HIV infection during the COVID-19 pandemic in Taiwan." Sexually Transmitted Infections, 2020, doi:https://doi.org/10.1136/ sextrans-2020-054802

78. De Miguel Buckley R, et al. Social distancing to combat COVID19 led to a marked decrease in food-borne infections and sexually transmitted diseases in Spain. Journal of Travel Medicine. 2020;27(8). https://doi.org/10.1093/jtm/taaa134.

79. Public Health Ontario. "Monthly infectious disease surveillance report" (n.d.) https://www.publichealthontario.ca/-/media/ documents/surveillance-reports/infectious/surveillance-reportinfectious-diseases-2020.pdf?la=en

80. Jenness SM, et al. Projected HIV and bacterial STI incidence following COVID-related sexual distancing and clinical service interruption. The Journal of Infectious Diseases. 2021. https:/ doi.org/10.1093/infdis/jiab051.

81. Public Health Agency of Canada. "Report on sexually transmitted infections in Canada, 2017" https://www.canada.ca/en/publichealth/services/publications/diseases-conditions/report-sexuallytransmitted-infections-canada-2017.html\#a6

82. Rompalo AM, et al. Clinical manifestations of early syphilis by HIV status and gender. Sex Transm Dis. 2001;28(3):158-65. https://doi.org/10.1097/00007435-200103000-00007.

83. Rebbapragada A, Kaul R. More than their sum in your parts: the mechanisms that underpin the mutually advantageous relationship between HIV and sexually transmitted infections. Drug Discovery Today: Disease Mechanisms. 2007;4(4):237-46. https://doi.org/ 10.1016/j.ddmec.2007.12.003.

84. Burchell AN, et al. Enhanced Syphilis Screening among HIVPositive Men (ESSAHM): a study protocol for a clinicrandomized trial with stepped wedge design. Implementation Science. 2015;11(1). https://doi.org/10.1186/s13012-016-0371-0.

85. MacKinnon, Kinnon R, et al. "Patient perspectives on the implementation of routinised syphilis screening with HIV viral load testing: qualitative process evaluation of the enhanced syphilis screening among HIV-positive men trial." 2020, doi:10.21203/ rs.3.rs-44050/v1.

86. Tuite AR, et al. Cost-effectiveness of enhanced syphilis screening among HIV-positive men who have sex with men: a microsimulation model. PLoS ONE. 2014;9(7). https://doi.org/ 10.1371/journal.pone.0101240.

87. Johns DR, et al. Alteration in the natural history of neurosyphilis by concurrent infection with the human immunodeficiency virus. New England Journal of Medicine. 1987;316(25):1569-72. https://doi.org/10.1056/nejm198706183162503.

88. Berry CD, et al. Neurologic relapse after benzathine penicillin therapy for secondary syphilis in a patient with HIV infection. New England Journal of Medicine. 1987;316(25):1587-9. https://doi.org/10.1056/nejm198706183162507.

89. Musher DM. Syphilis, neurosyphilis, penicillin, and AIDS. Journal of Infectious Diseases. 1991;163(6):1201-6. https://doi. org/10.1093/infdis/163.6.1201.

90. Flood JM, Weinstock HS, Guroy ME, Bayne L, Simon RP, Bolan G. Neurosyphilis during the AIDS epidemic, San Francisco, 1985- 
1992. Journal of Infectious Diseases. 1998;177(4):931-40. https:// doi.org/10.1086/515245.

91. Tomkins A, et al. Screening for asymptomatic neurosyphilis in HIV patients after treatment of early syphilis: an observational study. Sexually Transmitted Infections. 2017;94(5):337-9. https://doi.org/10.1136/sextrans-2016-052938.

92. Ghanem KG. Management of adult syphilis: key questions to inform the 2015 Centers for Disease Control and Prevention sexually transmitted diseases treatment guidelines: Table 1. Clinical Infectious Diseases. 61(suppl 8):2015. https://doi.org/10.1093/ $\mathrm{cid} / \mathrm{civ} 714$.

93. Marra CM, et al. Normalization of serum rapid plasma reagin titer predicts normalization of cerebrospinal fluid and clinical abnormalities after treatment of neurosyphilis. Clinical Infectious Diseases. 2008;47(7):893-9. https://doi.org/10.1086/591534.

94. Xiao Y, et al. Serological response predicts normalization of cerebrospinal fluid abnormalities at six months after treatment in HIV-negative neurosyphilis patients. Scientific Reports. 2017;7(1). https://doi.org/10.1038/s41598-017-10387-x.

95. Dunaway SB, et al. Neurosyphilis treatment outcomes after intravenous penicillin $G$ versus intramuscular procaine penicillin plus oral probenecid. Clinical Infectious Diseases. 2019;71(2):267-73. https://doi.org/10.1093/cid/ciz795.

96. Buitrago-Garcia D, et al. Antibiotic therapy for adults with neurosyphilis. Cochrane Database of Systematic Reviews. 2019. https://doi.org/10.1002/14651858.cd011399.pub2.

97. Klausner, Jeffery D., et al. Cefixime for alternative syphilis treatment. (n.d.) https://clinicaltrials.gov/ct2/show/NCT03660488

98. Causer LM, et al. An evaluation of a novel dual treponemal/ nontreponemal point-of-care test for syphilis as a tool to distinguish active from past treated infection. Clinical Infectious Diseases. 2015;61(2):184-91. https://doi.org/10.1093/cid/civ243.

99. Zhou C, et al. PCR detection for syphilis diagnosis: status and prospects. Journal of Clinical Laboratory Analysis. 2019;33(5). https://doi.org/10.1002/jcla.22890.

100. Grange PA, Gressier L, Dion PL, Farhi D, Benhaddou N, Gerhardt $\mathrm{P}$, et al. Evaluation of a PCR test for detection of Treponema Pallidum in swabs and blood. Journal of Clinical Microbiology. 2012;50(3):546-52. https://doi.org/10.1128/jcm.00702-11.
101. Molina J-M, et al. On demand post exposure prophylaxis with doxycycline for MSM enrolled in a prep trial. In: Conference on Retroviruses and Opportunistic Infections. Washington: Seattle; 2017.

102. Tattersall, Tessa Lawson, et al. "Preliminary results of the Dual Daily HIV and Syphilis Pre-Exposure Prophylaxis (DuDHS) trial." The 29th Annual Canadian Conference on HIV/AIDS Research, 2020.

103. Bolan RK, et al. Doxycycline prophylaxis to reduce incident syphilis among HIV-infected men who have sex with men who continue to engage in high-risk sex. Sexually Transmitted Diseases. $2015 ; 42(2): 98-103$. https://doi.org/10.1097/olq. 0000000000000216.

104. Steen R, et al. Periodic presumptive treatment of curable sexually transmitted infections among sex workers. AIDS. 2012;26(4): 437-45. https://doi.org/10.1097/qad.0b013e32834ed991.

105. Lithgow $\mathrm{KV}$, et al. A defined syphilis vaccine candidate inhibits dissemination of Treponema Pallidum Subspecies Pallidum. Nature Communications. 2017;8(1). https://doi.org/10.1038/ ncomms 14273.

106. Tan DH, et al. Canadian guideline on HIV pre-exposure prophylaxis and nonoccupational postexposure prophylaxis. Canadian Medical Association Journal. 2017;189(47). https://doi.org/10. 1503/cmaj.170494.

107. Centre for Disease Control and Prevention, "Preexposure prophylaxis for the prevention of HIV infection in the United States 2017 Update A Clinical Practice Guideline", https://www.cdc. gov/hiv/pdf/risk/prep/cdc-hiv-prep-guidelines-2017.pdf

108. Werner RN, et al. Incidence of sexually transmitted infections in men who have sex with men and who are at substantial risk of HIV infection - a meta-analysis of data from trials and observational studies of HIV pre-exposure prophylaxis. PLOS ONE. 2018;13(12). https://doi.org/10.1371/journal.pone.0208107.

109. Sexual Health London. "Home STI testing, regular and emergency contraception. 2021.’https://www.shl.uk/

Publisher's Note Springer Nature remains neutral with regard to jurisdictional claims in published maps and institutional affiliations. 